\title{
Hyperspectral Terahertz Tomography in Amplitude Contrast
}

DOI:

10.1109/JSEN.2018.2881917

\section{Document Version}

Accepted author manuscript

Link to publication record in Manchester Research Explorer

\section{Citation for published version (APA):}

Banuelos-Saucedo, M. A., \& Ozanyan, K. (2018). Hyperspectral Terahertz Tomography in Amplitude Contrast. IEEE Sensors Journal. https://doi.org/10.1109/JSEN.2018.2881917

\section{Published in:}

IEEE Sensors Journal

\section{Citing this paper}

Please note that where the full-text provided on Manchester Research Explorer is the Author Accepted Manuscript or Proof version this may differ from the final Published version. If citing, it is advised that you check and use the publisher's definitive version.

\section{General rights}

Copyright and moral rights for the publications made accessible in the Research Explorer are retained by the authors and/or other copyright owners and it is a condition of accessing publications that users recognise and abide by the legal requirements associated with these rights.

\section{Takedown policy}

If you believe that this document breaches copyright please refer to the University of Manchester's Takedown Procedures [http://man.ac.uk/04Y6Bo] or contact uml.scholarlycommunications@manchester.ac.uk providing relevant details, so we can investigate your claim.

\section{OPEN ACCESS}




\title{
Hyperspectral Terahertz Tomography in Amplitude Contrast
}

\author{
Miguel A. Banuelos-Saucedo and Krikor B. Ozanyan, Senior Member, IEEE
}

\begin{abstract}
Hyperspectral tomography in THz amplitude contrast is presented using a time-domain spectroscopy system in the spectral range $0.3-2.5 \mathrm{THz}$. The Fourier transformed signal data is used to reconstruct test objects' cross-sections at multiple frequencies using standard filtered backprojection. The full hyperspectral set of images reconstructed at around 300 adjacent spectral points is used to trace the combined contribution of BeerLambert volume attenuation, Fresnel reflection losses and Rayleigh roughness scattering losses, which is in good overall agreement with the experimental results. The image quality for Styrofoam (refractive index around 1.02, attenuation coefficient $<$ $1 \mathrm{~mm}^{-1}$ ) test objects is best in the range $0.8-2.0 \mathrm{THz}$ depending on the porosity of the material.
\end{abstract}

Index Terms - THz, hyperspectral imaging, hard-field tomography, Rayleigh roughness, time-domain spectroscopy.

\section{INTRODUCTION}

$\mathrm{T}$ $\mathrm{Hz}$ radiation has the ability to penetrate some dielectric materials such as paper, cardboard, ceramics, plastics and textiles [1]. This has motivated the development of imaging and tomography systems at these frequencies [2, 3]. Applications include spectroscopic identification and imaging, nondestructive evaluation, security inspection and defect detection, among others. Many $\mathrm{THz}$ systems require a bulky laser but, in search of practical applications, there has been a growing interest in developing $\mathrm{THz}$ portable systems [4,5]. Advances in technology have enabled the development of reduced footprint $\mathrm{THz}$ systems based on communications lasers around $1.5 \mu \mathrm{m}$. It is expected that continuous wave (CW) $\mathrm{THz}$ sources, such a quantum cascade laser (QCL) will have a greater role in the development of compact $\mathrm{THz}$ imaging systems.

Examples of $\mathrm{THz}$ tomography imaging have been reported to date using $\mathrm{THz}$ pulsed $[1,6-8]$, or continuous wave $(\mathrm{CW})$ sources [1, 9-11]. Results achieved by time-of-flight or phase contrast with pulsed sources are the most convincing yet. These methods employ the time domain characteristics of the signal and do not depend on the various mechanisms competing in the

Manuscript received September xx, 2018; accepted Xxxx xx, 2018. Date of publication $\mathrm{Xxxx} x x, 2018$; date of current version November 12, 2018. An earlier version of this paper was presented at the IEEE SENSORS 2014 Conference in Valencia, Spain, and was published in its Proceedings (doi: 10.1109/ICSENS.2014.6985244). This work was supported in part by the U.K. Engineering and Physical Sciences and in part by the Consejo Nacional de Ciencia y Tecnología (CONACyT, Mexico) together with Universidad Nacional Autonónoma de México. M.A. Bañuelos Saucedo is with Instituto de attenuation of the transmitted photon flux. However, with the progress in the $\mathrm{CW} \mathrm{THz} \mathrm{sources,} \mathrm{imaging} \mathrm{in} \mathrm{amplitude} \mathrm{contrast}$ is expected to rise to a greater relevance. $\mathrm{CW}$ narrowband systems will allow imaging in $\mathrm{THz}$ amplitude contrast at discrete wavelengths important for security and non-destructive testing. To achieve indirect imaging from the amplitude of the transmission beam hard-field tomography, it is also essential to understand and manage the soft-field scattered component in the transmitted $\mathrm{THz}$ signal; problems associated with $\mathrm{THz}$ computed tomography include Snell and Fresnel refraction [1215], and Gaussian THz beam profile [16]. The novelty in this work is that using a pulsed $\mathrm{THz}$ tomography system wide spectral information is accessed, over one order of magnitude in wavelength, to study the quality of the hard-field reconstructed images as a function of wavelength. This allows to infer the wavelength dependent scattering contributions and thus potentially the wavelength range where reliable hard-field imaging is still possible.

\section{EXPERIMENTAL SETUP}

The implemented system is based on a $\mathrm{THz}$ time-domain spectroscopy system (THz-TDS), built around a Ti:Sapphire laser (Coherent MIRA 900), pumped by a solid-state laser (Coherent Verdi V18). The Ti:Sapphire laser produces $150 \mathrm{fs}$ pulses@ $76 \mathrm{MHz}$ with $800 \mathrm{~nm}$ wavelength. A large aperture $(400 \mu \mathrm{m}) \mathrm{GaAs}$ photoconductive antenna, biased at $180 \mathrm{~V}$ DC and electronically modulated at $10 \mathrm{kHz}$, is used to generate the $\mathrm{THz}$ pulsed radiation. Detection is performed by electro-optic sampling in a $2 \mathrm{~mm}$ thick ZnTe crystal. A quarter-wave plate (Newport 10RP14-28), a Wollaston prism (Thorlabs WP10) and a balanced detector (Newport 2007) are used to analyze the signal. Demodulation is achieved by a lock-in amplifier (Signal Recovery 7265)[17].

The tomography setup (Fig. 1) utilizes two identical sets comprising a couple of metallic off-axis collimating and focusing parabolic mirrors $(\mathrm{EFL}=50.8 \mathrm{~mm}$ and $177 \mathrm{~mm})$, producing a $\mathrm{THz}$ beam waist that is used to probe the attenuation path integrals of the imaged object. A couple of 4

Ciencias Aplicadas y Tecnología, Universidad Nacional Autónoma de México. Circuito Exterior S/N, Ciudad Universitaria, A. P. 70-186, Delegación Coyoacán, C.P. 04510, México CdMx. México (e-mail: miguel.banuelos@icat.unam.mx).

Krikor B. Ozanyan is with the School of Electrical and Electronic Engineering, University of Manchester, Manchester, M13 9PL, U.K. (email: k.ozanyan@manchester.ac.uk).

Digital Object Identifier 10.1109/JSEN.2018.nnnnnn. 
$\mathrm{mm}$ iris diaphragms (apertures) are placed in front of and behind the object to suppress off-waist scattered photons and favor the detection of hard-field photons. Knife-edge test estimates indicate a beam waist diameter of $5 \mathrm{~mm} @ 0.586 \mathrm{THz}$ with an effective Rayleigh range of $67 \mathrm{~mm} @ 0.586 \mathrm{THz}$. Similar to [6], each tomography projection (i.e. a set of multiple parallel beams at a fixed angle) is acquired by moving the object across the $\mathrm{THz}$ beam waist using a linear translation stage. Projections at additional angles are generated by placing the imaged object on a rotational table. In the chosen configuration, the $\mathrm{THz}$ radiation incident to the test objects used is s-polarized.

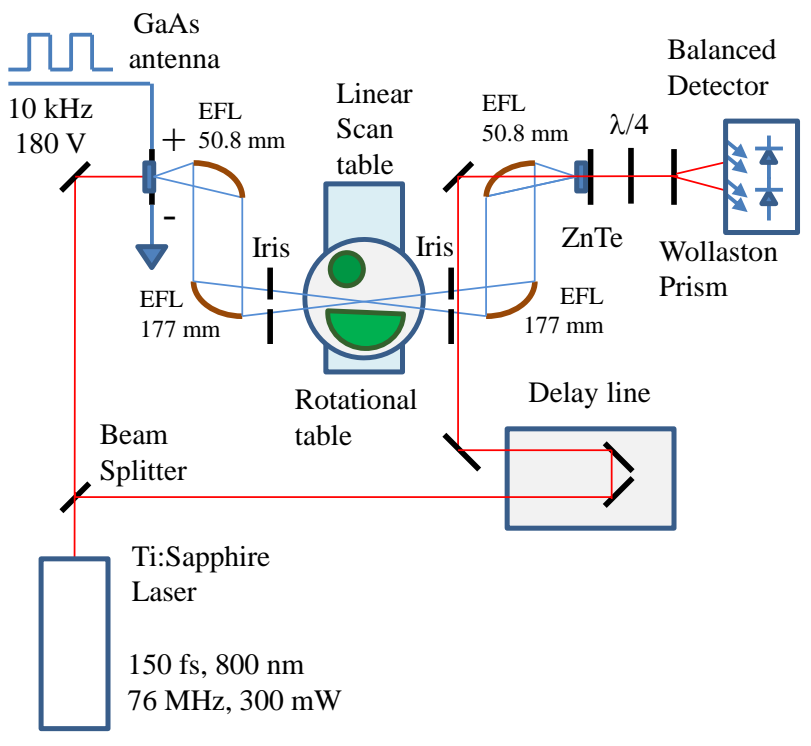

Fig. 1. Experimental setup of a THz time-domain spectroscopy system adapted for tomography.

Unavoidably, the iris diaphragms substantially reduce the signal, since they block a portion of the $\mathrm{THz}$ beam, thus affecting the noise performance of the system. The signal-tonoise ratio (SNR) of the system was estimated as

$$
S N R_{d B}=\log \frac{\text { mean amplitude }}{\text { standard deviation of amplitude }}
$$

for a set of ten consecutive measurements. The SNR of the system is plotted in Fig. 2. A minimum of $25 \mathrm{~dB}$ dynamic range is obtained in the operating range of $0.3 \mathrm{THz}$ to $2.5 \mathrm{THz}$. This threshold may limit the dynamic range of reconstructed images and it is set substantially below the common SNR figures found in the literature for THz-TDS systems (60 dB and above) due to the use of apertures imposing an order of magnitude amplitude reduction. Nevertheless, the obtained threshold is justified, as shown further, by the overall satisfactory quality of the $\mathrm{THz}$ images reconstructed in that frequency range. Above $2.5 \mathrm{THz}$, the response is limited by the finite bandwidth content of the signal pulse and frequency limitations of the electro-optic sampling performed with the ZnTe crystal.

This work takes advantage of the spectral information contained in the transmitted $\mathrm{THz}$ pulse. The access to a wide uninterrupted operating spectral range in THz-TDS allows a

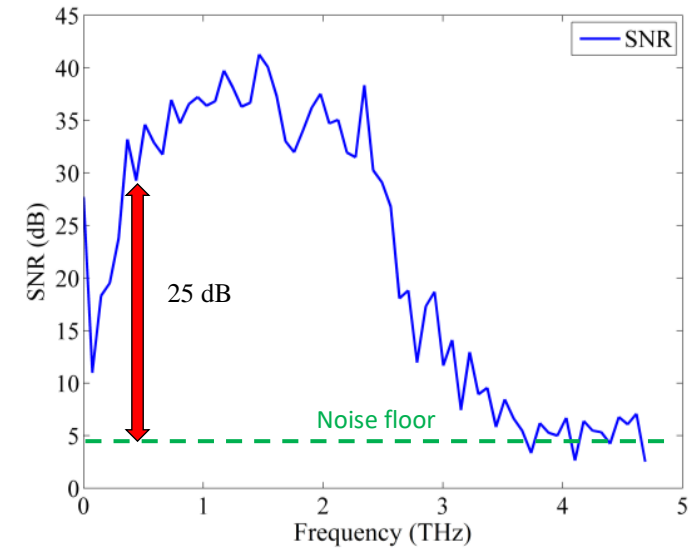

Fig.2. Signal-to-noise ratio of the $\mathrm{THz}$ tomography system.

"hyperspectral" approach to tomography imaging.

\section{HYPERSPECTRAL THZ TOMOGRAPHY}

THz-TDS typically operates at wavelengths between 0.12 $\mathrm{mm}$ and $1 \mathrm{~mm}$. Under these conditions, imaging of objects with sub-centimeter spatial detail may involve diffraction phenomena. One of the first reports on $\mathrm{THz}$ computed tomography based on a THz-TDS was presented by Ferguson et al. [18] in 2002. Reconstruction using diffraction tomography using pulsed $\mathrm{THz}$ sources has been covered in [6], and a review of $\mathrm{THz}$ tomography techniques is available in [1]. In practice, hard-field tomography (HFT) is easier to implement and may be able to provide faster access to important information of the analyzed object, if diffraction and scattering effects are understood and managed.

Imaging in time-of-flight contrast is the most straightforward tomography use of a TDS system. Nevertheless, the broadband frequency content of the THz source discussed in Section II, can be analyzed using a Fast Fourier Transform (FFT), and the amplitude can be used to reconstruct a separate image at each frequency component. This carries the inconvenience of sharing the signal power across the frequency components, but reconstruction can still be satisfactory with an appropriate SNR.

In typical spectroscopy applications it is common to acquire 1024 or 2048 sample points for each THz pulse. However, having complete flexibility in this regard, it was practical to reduce the number of points in order to shorten the total acquisition time at the cost of being able to reconstruct images at fewer frequencies. This is justified by the results obtained, as the set of images do not vary sharply with frequency. With this rationale, THz-TDS pulse signals were acquired at 128 sample points, zero padded to 1024. The application of FFT gives 512 frequency bins for the whole spectral range in Fig. 2, allowing to reconstruct amplitude contrast images at 350 different adjacent and equally spaced frequencies between $0.8 \mathrm{THz}$ and $2.5 \mathrm{THz}$.

Test objects ("phantoms") were scanned transversally using $1 \mathrm{~mm}$ steps, where each step allows the measurement of a path integral associated with a single projection beam (a single 
projection point). A set of 25 projections are acquired by rotating the object from $0^{\circ}$ to $180^{\circ}$ using $7.5^{\circ}$ increments. The reconstruction area is $65 \mathrm{~mm}$ by $65 \mathrm{~mm}$.

There are a range of materials suited for $\mathrm{THz}$ tomography study such as polypropylene, polyethylene, nylon and polyester [9]. For transmission measurements in particular, it is convenient to use materials with $\mathrm{THz}$ absorption coefficients between $0.1 \mathrm{~mm}^{-1}$ and $10 \mathrm{~mm}^{-1}$. In order to minimize refraction effects, a low THz refractive index is needed. These conditions are satisfied by Styrofoam, which was used to manufacture the phantoms used in this work.

The HFT approach is justified if refraction and scattering effects are suppressed. In the HFT framework, the Radon transform is applied to the projection data [19]

$$
p(\theta, t)=\iint f(x, y) \delta(x \cos \theta-y \sin \theta-t) d x d y
$$

where $\delta$ is the Dirac's delta function, $t$ varies in a direction orthogonal to the path integral direction, and $\theta$ is the projection angle. A standard MATLAB function (iradon) was used to

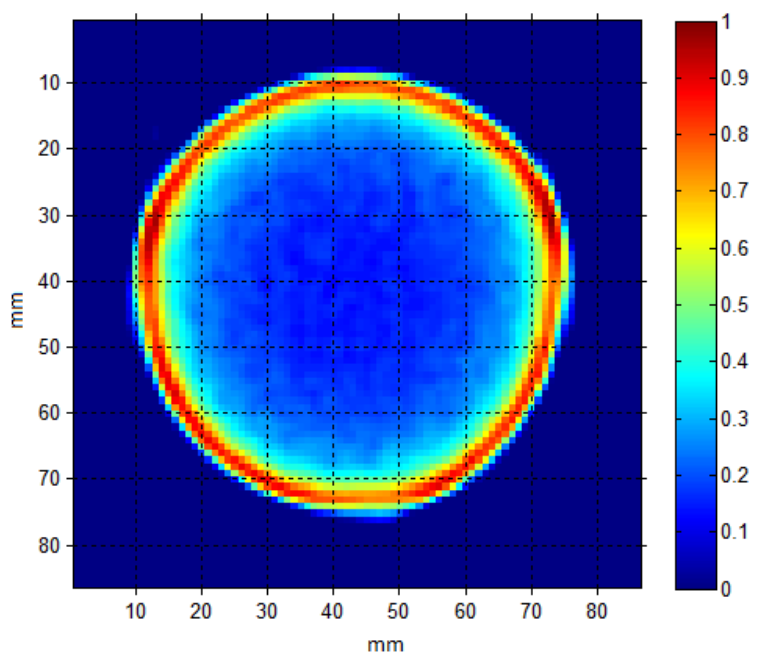

Fig. 3. Image reconstruction in normalized amplitude contrast of the crosssection of a $63 \mathrm{~mm}$ diameter Styrofoam cylinder at $1.992 \mathrm{THz}$.

reconstruct the images using filtered backprojection with linear interpolation and Hamming filtering [20].

Fig. 3 shows the image reconstruction with pixel size $1 \mathrm{~mm}$ $\mathrm{x} 1 \mathrm{~mm}$, of a $63 \mathrm{~mm}$ diameter Styrofoam cylinder cross section, using the signal amplitude at $1.992 \mathrm{THz}$. The shape of the cylinder is not an exact circle due to limitations on the tools used for making it. The reconstruction presents an apparent region of strong attenuation at the edge of the object while the inner volume appears almost transparent, despite the relatively long beam path. Image reconstructions of a set of four noncentrosymmetric Styrofoam phantoms of various more complex shapes are shown in Fig. 4.

The amplitude contrast reconstructions at several representative wavelengths/frequencies, along with the time-offlight contrast reconstructions [7], are shown in Table I. In amplitude contrast, all tested shapes manifest excessive attenuation at the edges. The contour fidelity is better at low frequencies: e.g. the cylindrical bore inside a cylindrical phantom is reconstructed at $0.586 \mathrm{THz}$ and $0.996 \mathrm{THz}$, but not at the higher frequencies $1.992 \mathrm{THz}$ and $2.520 \mathrm{THz}$.

It is notable that reconstructions in time-of-flight contrast (bottom of Table I) do not manifest excessive attenuation at the edges.

While all tomographic modalities are expected to provide some information on the inner structure of the object, amplitude contrast yields the spatial map of the attenuation coefficient of the material, whilst time-of-flight contrast yields a map of the refractive index. Overall, since the phantoms comprise of a single homogenous material, ideally, cross-section images of constant refractive index and constant attenuation coefficient should be expected. The apparent excessive attenuation at the phantom edges implies the involvement of additional surfacerelated loss mechanisms, potential candidates for which are identified, modelled and explained in the following section.

\section{ANALYSIS OF SURFACE LOSSES}

Surface effects are typically ignored in the application of the Radon transform HFT theory, e.g. in the case of x-rays for medical imaging, as their contribution is negligible. However, compared to $\mathrm{x}$-rays, the interpretation and use of tomography images in $\mathrm{THz}$ amplitude contrast require careful consideration of the surface-induced effects, due to the many orders of magnitude difference in the photon energy and wavelength.

\section{A. Styrofoam attenuation coefficient and refractive index}

According to the Beer-Lambert law, the $\mathrm{THz}$ beam amplitude is given by

$$
A=\sqrt{A_{0}^{2} e^{-\mu L}},
$$

where $A_{0}$ is the initial beam amplitude and $\mu$ is the attenuation coefficient averaged along path $\mathrm{L}$. The losses can be calculated using the path length through a cylinder

$$
L=2 \sqrt{r^{2}+x^{2}},
$$
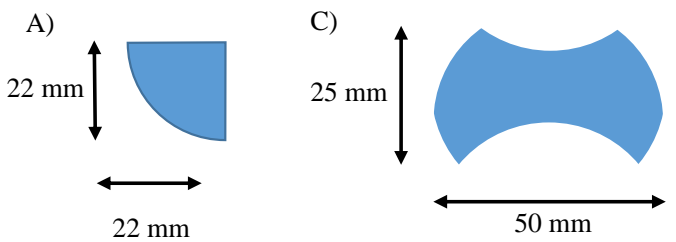

B)

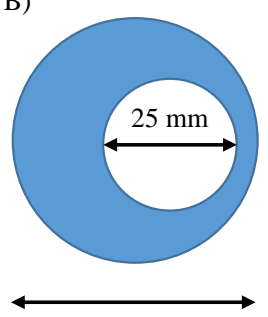

D)

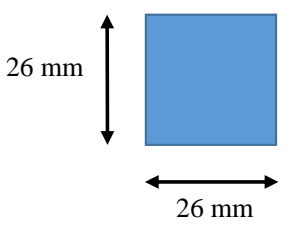

$50 \mathrm{~mm}$

Fig. 4. Set of Styrofoam phantoms. A) Quarter of cylinder. B) Cylinder with cylindrical bore. C) 'Xbox controller' shape. D) Square. 
TABLE I

IMAGE RECONSTRUCTION IN AMPLITUDE CONTRAST AND TIME-OF-FLIGHT OF FOUR STYROFOAM PHANTOMS

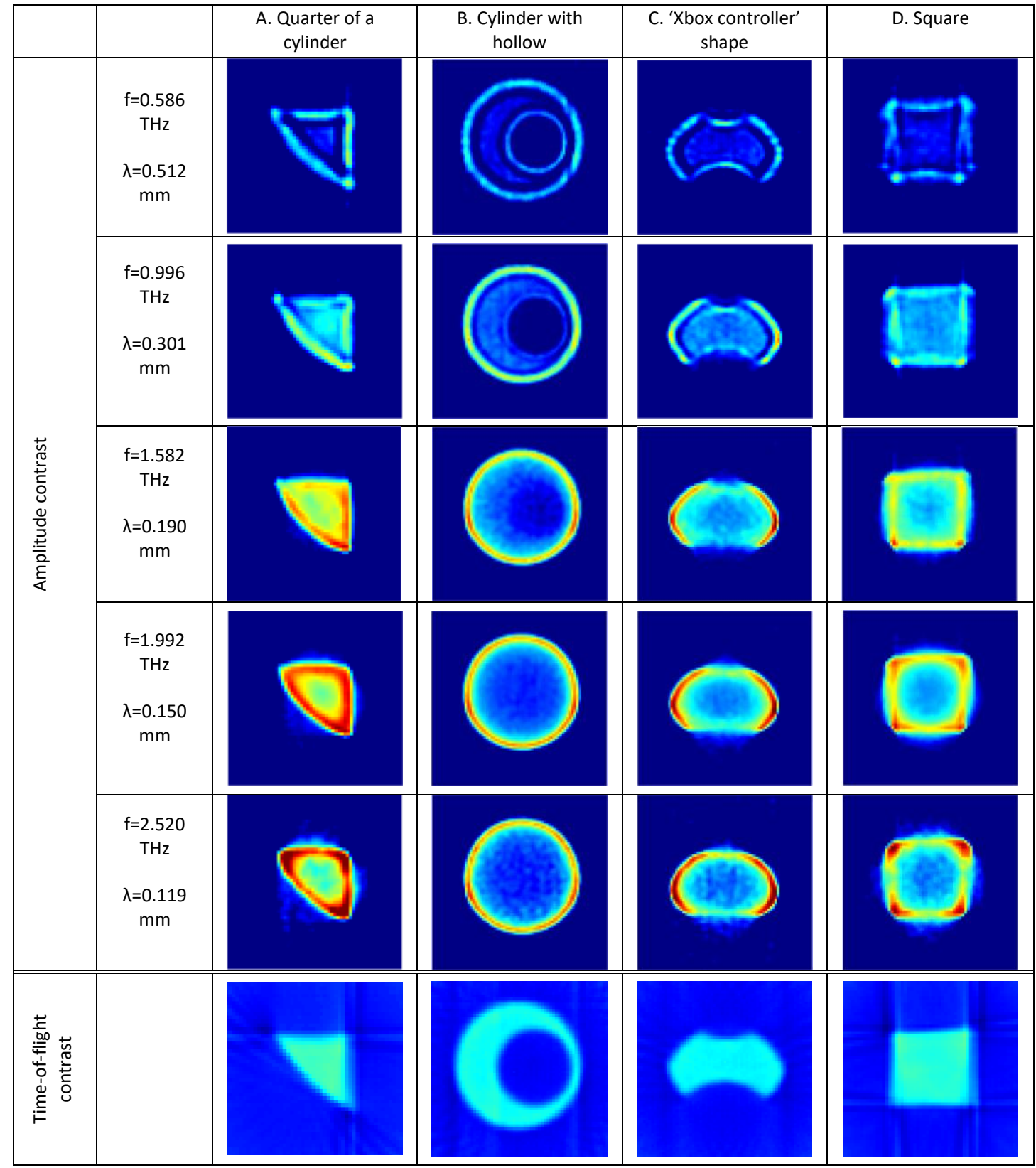

where $\mathrm{r}$ is the radius and $\mathrm{x}$ is the beam distance to the center of the cylinder.

Given two samples of different thickness, the attenuation coefficient can be estimated [21] using

$$
\mu(f)=\frac{-2 \ln \left(\frac{A_{2}(f)}{A_{1}(f)}\right)}{x_{2}-x_{1}},
$$

where $f$ is the frequency, $x_{1}$ and $x_{2}$ are the thicknesses of the samples and $A_{1}$ and $A_{2}$ are their amplitude responses, respectively. Using the same two samples, the refractive index is given by [21]

$$
n(f)=1+\frac{c\left(\Phi_{2}(f)-\Phi_{1}(f)\right)}{2 \pi f\left(x_{2}-x_{1}\right)}
$$

where $\Phi_{1}$ and $\Phi_{2}$ are the respective phase shifts as a function of frequency and $c$ is the speed of light in the sample.

The spectra of the attenuation coefficient and the refractive index for the $\mathrm{THz}$ working range from $0.2 \mathrm{THz}$ to $2.5 \mathrm{THz}$, are calculated using (4) and (5) in our case and plotted in Fig. 5. The refractive index can be considered constant over the range. A couple of small spikes can be observed at $1.099 \mathrm{THz}$ and $1.165 \mathrm{THz}$ which are due to water vapor [22]. They are attributed to the air supply line being fed through a drying cylinder, yielding $8 \%$ relative humidity inside the testing 


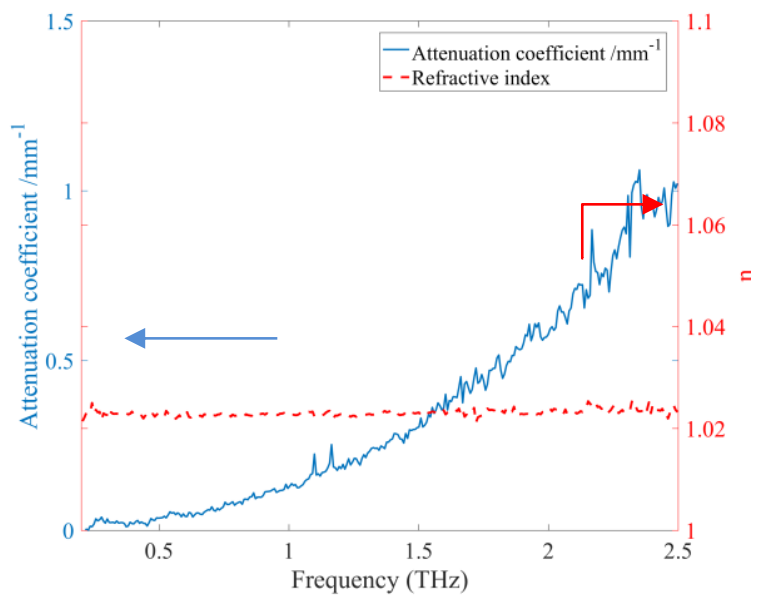

Fig. 5. Attenuation coefficient and refractive index of Styrofoam.

chamber. The spikes found above $2.0 \mathrm{THz}$ result from the noise as SNR degrades.

\section{B. Surface reflection}

According to the Fresnel equations for an s-polarized beam, as it is the case for the $\mathrm{THz}$ bean, incident at angle $\theta_{i}$, the transmittance from a perfect surface is:

$$
T_{s}=1-\left|\frac{n_{1} \cos \theta_{i}-n_{2} \sqrt{1-\left(\frac{n_{1}}{n_{2}} \sin \theta_{i}\right)^{2}}}{n_{1} \cos \theta_{i}+n_{2} \sqrt{1-\left(\frac{n_{1}}{n_{2}} \sin \theta_{i}\right)^{2}}}\right|^{2},
$$

where $n_{1}$ and $n_{2}$ are the refractive indices of the two media. Fig. 6 shows the effect of including the contribution of surface reflection losses, calculated from (6), to the overall attenuation. The Fresnel reflection losses produce a zone of apparent high attenuation at the edge of the circular cross-section, represented by the spikes at the two ends of the plot in Fig. 6. Since increased reflection results in reduced transmission, the reflection losses at the edge appear as attenuation in agreement with our observations in the amplitude contrast images. Since substantial reflection losses occur only at high $\theta$, their contribution is minimal at normal entry into the cylindrical

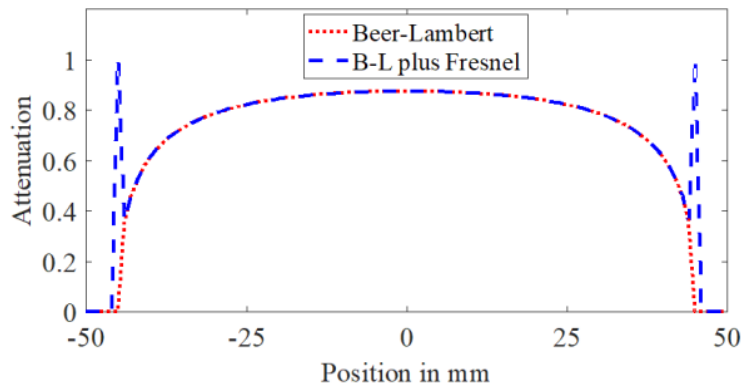

Fig. 6. Normalized attenuation losses due to Beer-Lambert law only and Beer-Lambert law plus Fresnel reflection, for a Styrofoam cylinder of 90 mm diameter@1 THz. phantom, in which case Beer-Lambert attenuation is dominating. The thickness of the high-attenuation ring at the periphery of the reconstructed object in Fig. 3 is seemingly larger than the width of the prominent Fresnel reflection losses, as indicated by the spikes in Fig. 6 . This can be a consequence of inadequate spatial resolution due to the geometry of the $\mathrm{THz}$ beam. However, other surface-related attenuation mechanisms, such as surface scattering, need to be considered as well.

\section{Surface scattering}

At $\mathrm{THz}$ frequencies, Styrofoam has a rough surface where

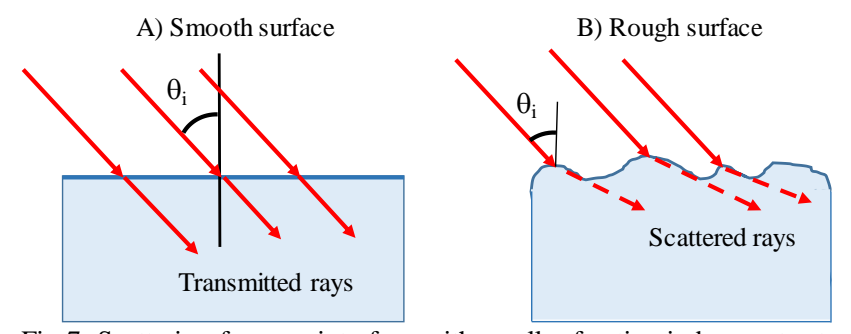

Fig 7. Scattering from an interface with small refractive index contrast. A) smooth surface, beams transmitted without notable deviation even at moderate incidence angles; B) rough surface, the angle of incidence is substantially increased for some beams.

incident beams will be arriving at a range of incidence angles, therefore producing scattered transmitted beams and reducing the total amount of detected photons (Fig. 7). For refractive index close to $1 \mathrm{It}$ is possible to model the Styrofoam surface scattering using the Rayleigh roughness factor[23]:

given

$$
\rho=e^{-g}
$$

$$
g=\left(\frac{4 \pi \sigma \cos \theta_{i}}{\lambda}\right)^{2},
$$

where $\sigma$ is the standard deviation of the surface roughness, $\theta_{i}$ is the beam angle of incidence, and $\lambda$ is the free space wavelength. The roughness factor $\rho$ represents the mean power scattered along the specular direction [23], dominating the signal at small values of $\mathrm{g}$. The diffuse scattered component, contributing to transmission loss caused by surface roughness, is dominating at large incidence angles when $g>>1$. In the case of tomography in amplitude contrast, the apparent transmission is modelled by correcting the Beer-Lambert attenuation for the contribution from $\rho$ :

$$
T_{m_{s}}=\rho T_{s}
$$

where $T m_{s}$ is the modified transmittance for an s-polarized beam, $T_{s}$ is the Fresnel transmittance in the absence of roughness given by (6) and $\rho$ is the Rayleigh roughness factor given by (7). To model the transmission losses through a surface with non-negligible roughness, the effects of the beam scattering given by (9) are combined to the Beer-Lambert attenuation and Fresnel reflection losses. 
TABLE II

NORMALIZED ATTENUATION PROFILE.ALONG THE DIAMETER OF A CYLINDRICAL PHANTOM AND RECONSTRUCTED IMAGES FOR TWO STYROFOAM MATERIALS, AT 1 THZ. FRESNEL AND FRESNEL PLUS SURFACE SCATTERING PLOTS ARE ARBITRARILY SHIFTED By 0.25 AND 0.5 RESPECTIVELY.

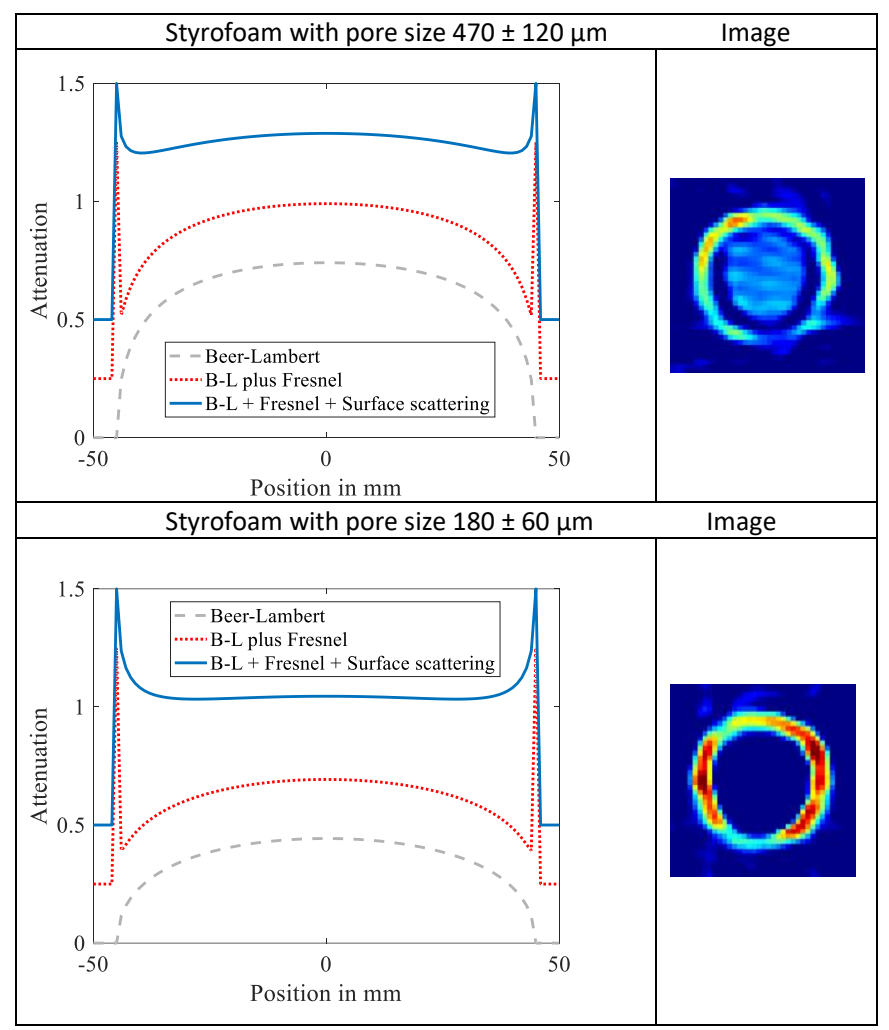

To compare the cumulative contribution of the three mechanisms with experimental images, calculations and tomography measurements were undertaken with cylindrical phantoms of identical diameters, cut from two Styrofoam materials of different porosity $(180 \pm 60 \mu \mathrm{m}$ and $470 \pm 120 \mu \mathrm{m}$, respectively, as established by microphotography and image processing). Using (4), (6) and (9), the calculated attenuation profiles and the images reconstructed from experimental data, at $1 \mathrm{THz}$, are presented in Table II. The profile model shows enhanced edge attenuation because of high reflectance at near grazing incidence. The contribution of surface roughness is more prominent at smaller $\sigma$, in agreement with (8).

Further, the cumulative contribution of the three transmission loss mechanisms was modelled, for the larger porosity cylindrical phantom, at three different $\mathrm{THz}$ wavelengths. Using (4), (6) and (9), the modelled profiles and the experimental images are shown in Table III. Higher frequencies notably suppress the influence of surface roughness, as expected from (7), (8) and (9): the surface roughness losses should decrease exponentially with the square of the frequency. Volume attenuation gains prominence with frequency as implied by both, the simulated attenuation profile and the images reconstructed from experimental data. In the reconstructed images, the thickness of the high attenuation edge zone does not decrease with frequency as strongly as the modelling implies. This may indicate the limitations of applying the random rough
TABLE III

NORMALIZED ATtENUATION PROFILE ALONG THE DiAMETER OF A CYLINDRICAL PHANTOM OF LARGE POROSITY STYROFOAM AND RECONSTRUCTED IMAGES AT 3 DIFFERENT THZ FREQUENCIES. FRESNEL AND FRESNEL PLUS SURFACE SCATTERING PlOTS ARE ARBITRARILY SHIFTED BY 0.25 AND 0.5 RESPECTIVELY.

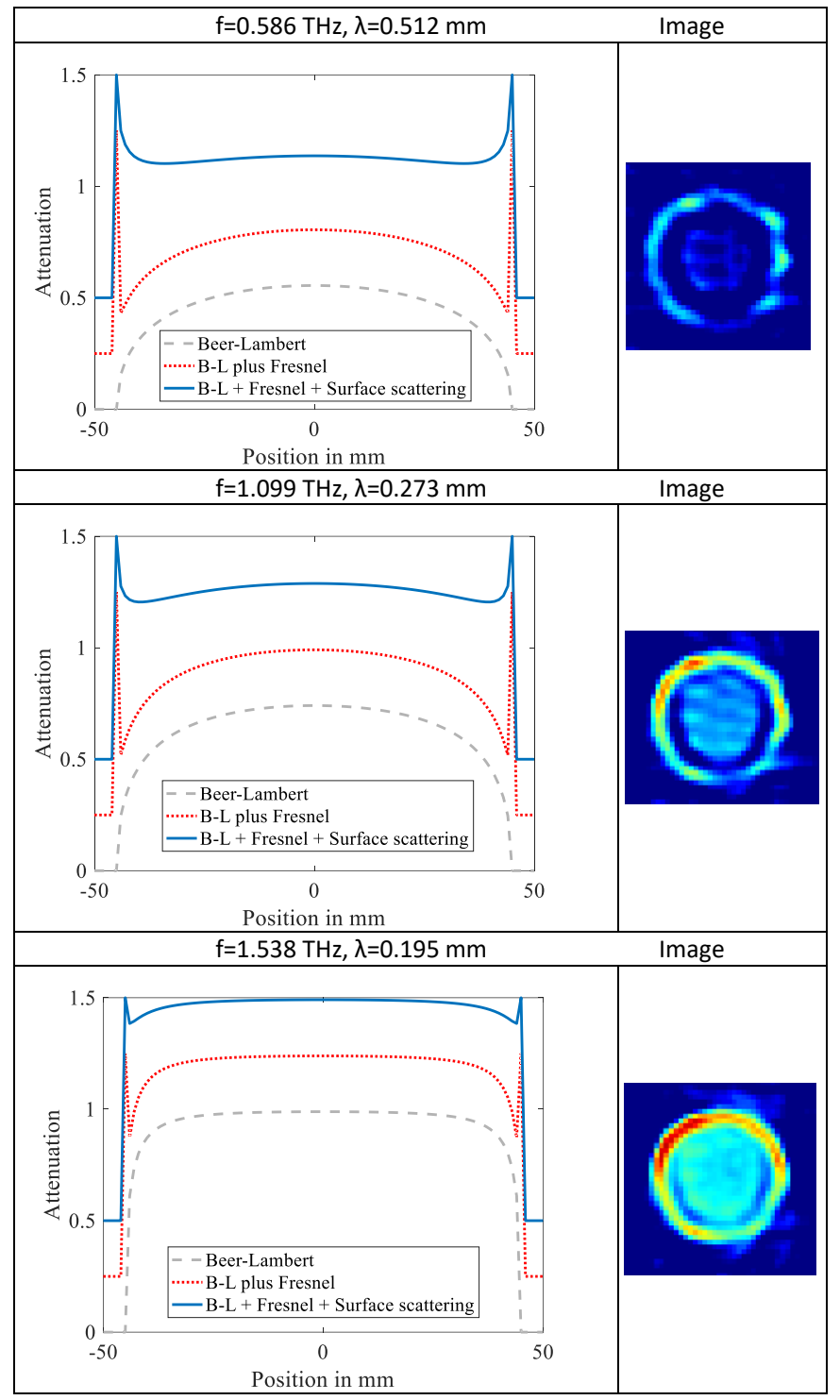

surface approach (7) (8) to our THz transmission model (9). In HFT based purely on the Beer-Lambert attenuation (dashed line plots), the excessive attenuation apparent at the edges is considered as an artefact. The latter, however, if well understood and managed, can be useful in $\mathrm{THz}$ imaging applications where emphasizing the contour of an object is a priority.

The demonstrated hyperspectral $\mathrm{THz}$ tomography capability was further applied to two composite phantoms, with identical geometry shown in Fig. 8, cut from the two Styrofoam materials of different porosity. Although such composite phantoms are closer to realistic imaging scenarios, the modelling of transmission losses with account to surface roughness becomes prohibitively complicated, because of the large variation in incidence angles: a) at beam entry and beam exit for each constituent object; b) spread in incidence angles within the 


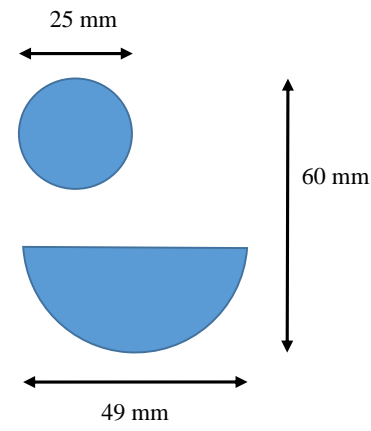

Fig. 8. Composite Styrofoam phantom: a circle and half-moon, similar to the phantom used in [7] for time-of-flight THz tomography.

beam waist for small size objects or features; $c$ ) variation across the different parallel beams at each projection angle. Therefore, only the image reconstructions are presented in Table IV. Overall, the phantom geometry is reproduced with reasonable fidelity. Although at low $\mathrm{THz}$ frequencies Styrofoam is transparent, the phantom contour is discernible at frequencies as low as $0.234 \mathrm{THz}$. The contour becomes more accentuated as the frequency increases, indicating a strong attenuation at the edges, in accord with the Rayleigh-Rice perturbation theory [24]. Furthermore, at higher frequencies, the inner region of the constituent objects becomes more visible, in accordance with the frequency response of the Beer-Lambert attenuation (Fig. 5). Efficient enhancement of surface roughness losses is expected at higher frequencies according to (9), however, implicit to the model is that the free space wavelength $\lambda$ is small enough compared to $\sigma$. This is not the case within the whole of the hyperspectral span studied here. Indeed, at the largest wavelengths the effect of roughness due to the pores will be weak, while much coarser roughness, induced by the imperfect cutting of the shapes, is likely to affect almost the whole of the hyperspectral span.

Table IV is a small excerpt from the complete hyperspectral set of around 350 images of each object, since the available spectral resolution is much finer than the spectral scale over which image properties are observed to vary. At some of the frequencies the quality of imaging is affected by excessive noise and/or atmospheric absorption. The complete hyperspectral sets of tomography images are available, as animated image sequence files attached to this paper, for the composite phantom (Fig. 8) cut from the two porosity materials <composite 180 um.avi> and <composite 470 um.avi>.

\section{CONCLUSION}

The frameworks of THz-TDS and HFT are combined to generate hyperspectral tomography images of test objects, in the range $0.3-2.5 \mathrm{THz}$, in amplitude attenuation contrast. Depending on the shape and surface roughness of the object, the HFT path attenuation contribution can be weaker than attenuation due to surface losses and for some wavelengths the object contour becomes the most prominent reconstructed feature. This can be useful in $\mathrm{THz}$ imaging applications where capturing the contour of an object is a priority.

Modelling of cross-section transmission loss profiles,
TABLE IV:

IMAGE RECONSTRUCTION FOR TWO PHANTOMS OF IDENTICAL CROSSSECTIONS MADE OF TWO STYROFOAM MATERIALS

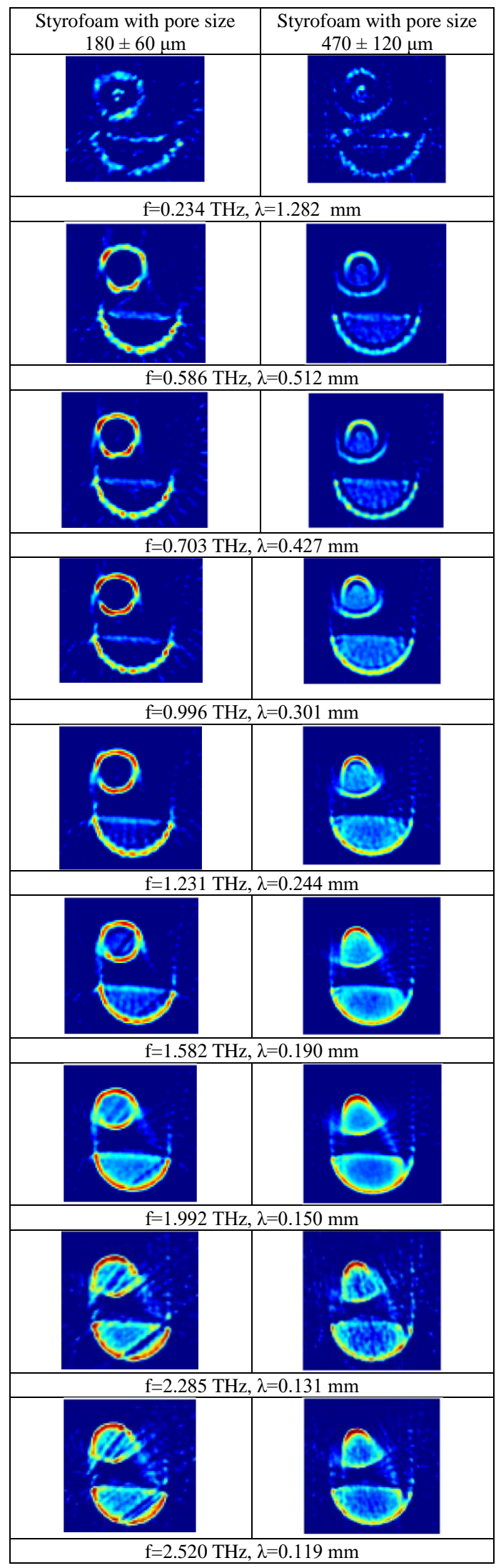


sensitive to wavelength and object geometry, combining BeerLambert volume attenuation, Fresnel reflection losses and roughness scattering losses, are in good overall agreement with the experimental results for a cylindrical object. For more complex and composite objects, the contour is reconstructed reasonably well over the whole hyperspectral range, but volume attenuation becomes prominent only in a limited frequency band. An "optimal" frequency for reconstruction would be one with high dynamic range and low scattering, for example, $\mathrm{f}=0.996 \mathrm{THz}$.

In addition to motivating the development of narrowband THz systems targeting particular objects, the hyperspectral $\mathrm{THz}$ tomography approach allows the translation of tomography methods and solutions developed theoretically, but difficult to implement experimentally at shorter wavelengths, such as the IR. An example is secondary multi-parameter contrast imaging based on spectral fitting[25]. This requires attenuation contrast images at a substantial number of spectral points, which is easier to achieve by TDS in the THz.

\section{REFERENCES}

[1] J. P. Guillet et al., "Review of Terahertz Tomography Techniques," (in English), Journal of Infrared, Millimeter, and Terahertz Waves, vol. 35, no. 4, pp. 382-411, 2014.

[2] S. P. Mickan and X. C. Zhang, "T-ray sensing and imaging," International Journal of High Speed Electronics and Systems, vol. 13, no. 2, pp. 601-676, 2003.

[3] Y. S. Lee, Principles of Terahertz Science and Technology. Springer-Verlag, 2009.

[4] D. Fratzscher, J. Beckmann, L. S. v. Chranowski, and U. Ewert, "Computed THz Tomography," in 18th World Conference on Nondestructive Testing, Durban, South Africa, 2012, p. 10: South African Institute for Non-Destructive Testing (SAINT)

[5] A. Malinowski et al., "Fiber MOPA based tunable source for terahertz spectroscopy," Laser Physics Letters, vol. 9, no. 5, pp. 350354, 2012.

[6] S. Wang and X. C. Zhang, "Pulsed terahertz tomography," Journal of Physics D: Applied Physics, vol. 37, no. 4, p. R1, 2004.

[7] K. B. Ozanyan, P. Wright, M. R. Stringer, and R. E. Miles, "Hardfield THz tomography," IEEE Sensors Journal, vol. 11, no. 10, pp. 2507-2513, 2011.

[8] J. B. Perraud et al., "Terahertz imaging and tomography as efficient instruments for testing polymer additive manufacturing objects," Applied Optics, vol. 55, no. 13, pp. 3462-3467, 2016.

[9] K. L. Nguyen et al., "Three-dimensional imaging with a terahertz quantum cascade laser," Optics Express, vol. 14, no. 6, pp. 21232129, 2006.

[10] G. C. Trichopoulos and K. Sertel, "Broadband Terahertz Computed Tomography Using a 5k-pixel Real-time THz Camera," Journal of Infrared, Millimeter, and Terahertz. Waves, journal article vol. 36, no. 7, pp. 675-686, 2015.

[11] B. Recur et al., "Investigation on reconstruction methods applied to 3D terahertz computed tomography," Optics Express, vol. 19, no. 6, pp. 5105-5117, 2011.

[12] S. Mukherjee, J. Federici, P. Lopes, and M. Cabral, "Elimination of Fresnel Reflection Boundary Effects and Beam Steering in Pulsed Terahertz Computed Tomography," Journal of Infrared, Millimeter, and Terahertz Waves, journal article vol. 34, no. 9, pp. 539-555, 2013.

[13] J. Tepe, T. Schuster, and B. Littau, "A modified algebraic reconstruction technique taking refraction into account with an application in terahertz tomography," Inverse Problems in Science and Engineering, vol. 25, no. 10, pp. 1448-1473, 2017.

[14] A. Brahm, A. Wilms, M. Tymoshchuk, C. Grossmann, G. Notni, and A. Tünnermann, "Optical Effects at projection measurements for Terahertz tomography," Optics and Laser Technology, vol. 62, pp. 49-57, 2014.
[15] E. Abraham, A. Younus, C. Aguerre, P. Desbarats, and P. Mounaix, "Refraction losses in terahertz computed tomography," Optics Communications, vol. 283, no. 10, pp. 2050-2055, 2010.

[16] B. Recur et al., "Propagation beam consideration for 3D THz computed tomography," Optics Express, vol. 20, no. 6, pp. 5817$5829,2012$.

[17] M. Banuelos-Saucedo, "Signal and data processing for Terahertz imaging," PhD Thesis, Faculty of Physical Sciences, The University of Manchester, Manchester, UK, 2014.

[18] B. Ferguson, S. Wang, D. Gray, D. Abbot, and X. C. Zhang, "T-ray computed tomography," Opt. Lett., vol. 27, no. 15, pp. 1312-1314, 2002.

[19] A. C. Kak and M. Slaney, Principles of computerized tomographic imaging. Society for Industrial and Applied Mathematics, 2001.

[20] P. e. Grangeat, Tomography. Wiley, 2010. and A. G. Davies, "An investigation of beam focusing perturbations in THz spectroscopy measurements," in IRMMW-THz 2005. The Joint 30th International Conference on, 2005, vol. 2, pp. 421-422 vol. 2.

[22] M. v. Exter, C. Fattinger, and D. Grischkowsky, "Terahertz timedomain spectroscopy of water vapor," Opt. Lett., vol. 14, no. 20, pp. 1128-1130, 1989.

[23] P. Beckmann and A. Spizzichino, The Scattering of Electromagnetic Waves from Rough Surfaces. Artech House, 1987.

[24] R. Piesiewicz, C. Jansen, D. Mittleman, T. Kleine-Ostmann, M. Koch, and T. Kürner, "Scattering analysis for the modeling of THz communication systems," IEEE Transactions on Antennas and Propagation, vol. 55, no. 11 I, pp. 3002-3009, 2007.

[25] M. P. Wood and K. B. Ozanyan, "Simultaneous Temperature, Concentration, and Pressure Imaging of Water Vapor in a Turbine Engine," IEEE Sensors Journal, vol. 15, no. 1, pp. 545-551, 2015.

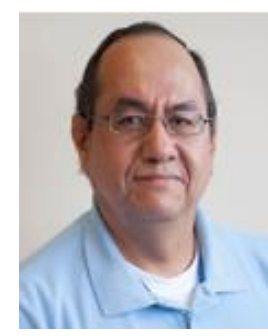

Miguel Angel Bañuelos Saucedo received MSc degree in Electrical Engineering from the Universidad Nacional Autónoma de México (UNAM) in 1997. Since 1994, he has been working at the Instituto de Ciencias Aplicadas y Tecnología (UNAM). He was the Head of the Electronics Group from 1999 to 2007. In 2010, he was granted a leave for studying his $\mathrm{PhD}$ at the University of Manchester, where he graduated in 2014. His current interests are biomedical systems and instrumentation in $\mathrm{THz}$ spectroscopy and tomography.

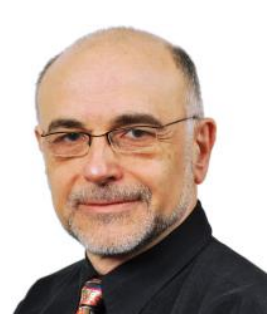

Krikor B. Ozanyan received the M.Sc. degree in engineering physics (semiconductors) and the $\mathrm{PhD}$ degree in solid-state physics in 1980 and 1989, respectively. He has more than 300 publications in the areas of devices, materials and systems for sensing and imaging. He is currently Director of Research at the School of EEE, at the University of Manchester, U.K. He is a Fellow of the Institute of Engineering and Technology, U.K, and the Institute of Physics, U.K. He was a Distinguished Lecturer of the IEEE Sensors Council in 2009 and 2010, and Guest Editor for the 10th Anniversary Issue of IEEE Sensors Journal in 2010, as well as the Special Issues on Sensors for Industrial Process Tomography in 2005 and THz Sensing: Materials, Devices and Systems in 2012. He was Editor-in-Chief of the IEEE Sensors Journal (2011- 2018) and was General Co-Chair of the IEEE SENSORS 2017. 